\title{
Design Considerations for 5G Mobile Network
}

\author{
Mehedi Shams Rony \\ University of Wales \\ King Edward VII Avenue \\ Cathays Park, Cardiff, CF10 3NS, UK
}

\author{
Adrian J Pullin \\ Resource Development International (RDI) Ltd \\ Midland Management Centre \\ 1A Brandon Lane, Coventry, CV3 3RD, UK
}

\begin{abstract}
With the advent of mobile technologies, communication is no longer a stationary element. In addition, with gradual sophistication of the technologies, the need for communication is no longer confined to voice only. Now, mobile communication encompasses voice, video and live streaming while the host is on the move. Various researches are going on to satisfy these needs to appreciable extent which can be taken farther to a satisfactory extent. Recently, 4G has been launched to address contemporary shortcomings of the mobile technology. To take the renovations steps ahead, the next phase of development will be commencing soon which is unofficially named as $5 \mathrm{G}$. In this paper, we address some of the shortcomings still persisting in the current stage of networking and focus on some design considerations of $5 \mathrm{G}$ including High Altitude Platform, Femtocells, and Cognitive Radio.
\end{abstract}

\section{General Terms}

Mobile network.

\section{Keywords}

High Altitude Platform, Femtocell, Cognitive Radio.

\section{INTRODUCTION}

The Internet is perhaps the most intensive and pervasive phenomenon at present. With sophisticated evolution of networking elements over so long a time, it has changed the way of pursuing knowledge, communication and collaboration. With passage of time, the Internet became an ultimate means for multimedia sharing including VOIP, video-conferencing, and live-video streaming. In most recent times, people started to demand such bandwidth-hungry applications while they are on move, taking these craving-formore one step further from what can be addressed by contemporary networking elements including algorithms, topologies, and protocols

Historically it was IPv4 that defined the standards for Internet communication. The fact that this protocol has been successfully serving the Internet communication for over 30 years of inception definitely claims accolade to the designers. However, like necessity is the mother of invention, the growing need from more and more users led to development of novel approaches and algorithms. Now mobility needs to be addressed in all spheres of developments. All concurrent renovations and inventions should consider that the user is no longer a stationary element. Most of the demands are for high QoS (Quality of Service) and ubiquitous connections [1] where $\mathrm{QoS}$ is a set of requirements that provides delay and jitter (i.e., delay variation). Some metrics for QoS are bandwidth, delay types, jitter, packet loss and reordering [2], that eventually resulted in different generations of developments including cellular networking.
This paper is organized as follows: Section 2 provides a brief history of the Internet and mobile networking developments. Femtocells, HAPs and cognitive radio are discussed in $3^{\text {rd }}$ Section 4. Section 4 provides a critical evaluation of the three promising concepts for $5 \mathrm{G}$ described. Section 5 discusses future research areas. Concluding remarks are given in Section 6.

\section{HISTORY}

It all started with ARPANET that commenced a research on data communication and the concept of Internet emerged. Protocols such as TCP/IP, FTP, and IPv4 started to evolve and get standardized. TCP/IP was designed to work with 7-layer OSI (Open Systems Interconnection) model which spawned subsequent layer-specific protocols. For instance, HTTP, FTP, SMTP were developed to work with application layer, TCP and UDP were created to work with transport layer, IPv4 was designed to address machines through network layer, and physical layer was designed to deal with numerous networking elements including algorithms, connectivity, and spectrum management and sharing.

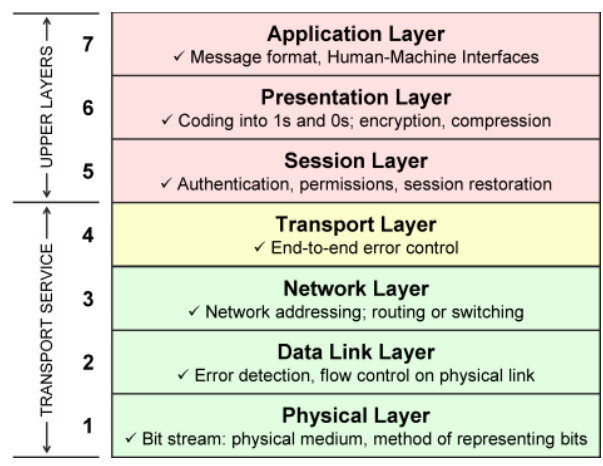

Fig 1: 7-Layer OSI Model [27]

The transport layer protocol IPv4 has been serving as a packet transmission protocol for over 30 years. IPv4 uses different header information along with a 4-byte addressing mechanism. The address space has already exhausted in February 2011 [3]; any new address to be allocated needs to resort to IPv6. Some unplanned allocation of IPv4 addresses might have been made better to support addressing of new hosts for a few more years. Like 127.x.x.x addresses that are used as loopback are wasting $\left(8 * 2^{16}\right)=16,777,216$ addresses. Additionally, the same number of addresses is simply wasted by each of the allocated companies owning the class-A addresses at the initial phase of class-based addressing.

IPv6 was introduced to address some of the limitations of IPv4. With its 16-byte address field in the packet header, it can literally address billions of millions of hosts. With better header design, it improvises the difficulties of IPv4 with 
regards to routing overhead, data streaming, and authenticated security.

The transition from IPv4 to IPv6 is gradually taking place. However, until that is fully established, IPv4 and IPv6 coexist. The conversation between them is done through some techniques like tunneling, encapsulation, and address translation.

With the advent of mobile technology and their promising communication features, these existing protocols needed renovations. MIP (Mobile Internet Protocol) was proposed to assist rapidly expanding mobile users with communication $[4$, 5, 6]. On the other hand, mobile protocols MIPv4 and MIPv6 were developed which correspond to IPv4 and IPv6. A mobile network works like tessellating hexagonal cells where each mobile tower is located at the points which transmit in three directions and can cover $360^{\circ}$ from that point

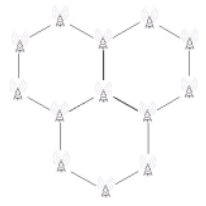

\section{Fig 2: Antenna locations in tessellating hexagonal cells.}

MIP works very simply but requires comprehensive connection mechanisms. The mobile host moves between subnets and is registered with HA (Home Agent) and FA (Foreign Agent). The communication is achieved with a tunnel (communication channel) between the agents.

Among the major problems associated with MIPv4 is the triangle routing problem where the sender sends data to a receiver through an HA instead of directly sending the packets to the receiver [7]. Besides, there are many other problems associated with address space exhaustion, lack of security, mobility, and data streaming.

RSVP (Resource reSerVation Protocol) is a QoS-aware and receiver-initiated protocol that requests the routers to reserve resources during the communication with sender. Some problems associated with this protocol for implementing in MIP such as pre-reservation, tunnelling, path identification, and mobile proxy are identified [1].

To offer uninterrupted RSVP QoS in MIP, there is a prereservation mechanism that reserves the resources in advance in every possible subnet the mobile host might travel. However, in most cases, this is a waste of resources. Also in the case of routers, the resources cannot be established in them along the tunnel. This is because RSVP messages are wrapped in packets; routers merely see packets, but cannot see the RSVP request inside. This was reported as the tunnelling problem [1]. With the advent of MIPv6, the tunnelling overhead is removed with the direct addressing of the mobile host in the foreign subnet [8]

When the mobile host enters a new subnet, the existing RSVP needs to be rescheduled for the whole path. This might actually be a waste because the new path will likely use the most of its old path- which is known as the path identification problem. There is another problem called the mobile proxy problem wherethe periodic refresh of the RSVP states decrease the battery life of the mobile host and a proxy and a proxy is required for a solution. Modulation techniques like
CDMA (Code Division Multiple Access) have been developed for transmission of multiple radio signals in a single channel. Unlike its counterpart TDMA (Time Division Multiple Access) which modulates the signal with time division technique, or FDMA (Frequency Division Multiple Access) which accommodates multiple signals in different frequency bands, the CDMA uses a transmitter-code and spread-spectrum technology to address this issue. Spreadspectrum is a technique to widen a signal to fit in a specific frequency domain. UMTS or CDMA2000 shifts the switching technology from circuit switching to packet switching.

WCDMA (Wideband Code Division Multiple Access) is an air-interface standard for $3 \mathrm{G}$ based on its predecessor CDMA It uses some additional techniques and duplexing to achieve higher speed with raised capacity to accommodate more users. As a further refinement of WCDMA, the HSDPA (High Speed Downlink Packet Access) protocol was developed that further enhances the data rate and throughput, and at the same time provides faster connection and shorter round trip times [9].

Generations of technology development have been involved in cellular systems. The steps towards mobile technology evolvement are defined as G-timelines. The beginning was with $1 \mathrm{G}$ in Tokyo, Japan in 1979. Then came GSM (Groupe Special Mobile) that was developed and termed as $2 \mathrm{G}$ in early 1990's with new promises on SMS technology, digital signal processing, and out-of-band phone-to-networking facilities.

The shift from voice-aware $2 \mathrm{G}$ to data-aware $3 \mathrm{G}$ was bridged by the $2.5 \mathrm{G}$ GPRS system which provides packet switching over existing GSM network [10]. GPRS can provide 171.2 kbps theoretically and uses a technology called MSC (Multi Slot Capability). It supports WAP (Wireless Application Protocol) and MMS (Multimedia Messaging Service). Some years later $2.75 \mathrm{G}$ was developed with EDGE (Enhanced Datarates for GSM Evolution) technology that used phase-shiftkeying to accommodate more speed.

$3 \mathrm{G}$ was developed with packet switching instead of circuit switching to support greater bandwidth ranging from $400 \mathrm{kbps}$ to $4000 \mathrm{kbps}$ [11]. The major problem associated with $3 \mathrm{G}$ is a mixture of technology; $3 \mathrm{G}$ utilizes different protocols for communication and this adds routing overhead for processing and translating the protocols. Another major problem is inflexible resource allocation where resources are preallocated before a $3 \mathrm{G}$ connection takes place and any idle resource is simply wasted. It is worthwhile mentioning that the infrastructure requirement for $3 \mathrm{G}$ is still in development phase for most part of the world which is similar to the recentmost $4 \mathrm{G}$ standard as well.

$4 \mathrm{G}$ is just at its inception in the mobile communication world. 4G comes with more data rates, especially brilliant for streaming videos, and resolves the resource allocation inflexibility of $3 \mathrm{G}$ to a little extent. $4 \mathrm{G}$ is provided by WiMAX (Worldwide Interoperability for Microwave Access) which provides much wider bandwidth through OFDMA (Orthogonal Frequency-Division Multiple Access). WiMAX is a connection-oriented protocol where connection is controlled by a base station. It provides a number of QoS for live-streaming. WiMAX may operate in unlicensed frequency bands and hence is vulnerable to interference and coexistence with other systems [12]. It also provides data encryption through AES (Advanced Encryption Standard) and DES (Data Encryption Standard). 
To bridge the transition from $3 \mathrm{G}$ to $4 \mathrm{G}$, the LTE (Long Term Evolution) has been proposed by 3GPP (3rd Generation Partnership Project). LTE re-engineers the OFDMA to generate a high-speed radio interface, and also includes EPC (Evolved Packet Core) which is an all-IP core network, and SON (Self-Organizing Network) which a significant part of LTE that can configure, optimize, and heal the connections itself [13].

Both WiMAX and LTE use OFDMA for signals where OFDMA has its own problem with single-cell and multiplecell system $[14,15]$. For rate controlling of OFDMA, it uses a special technique called Link adaptation.

An LTE system divides the spectrum into RBs (Resource Blocks) which are the smallest assignable resource unit [16]. If periodic reports from a UE (User Equipment) ascertain interference problem with the allocated RB, then some other $\mathrm{RB}$ can be randomly re-allocated to the UE. This solution is often successful in low-traffic and less dense situations. However, for heavier traffic, a co-ordinated approach is preferred; otherwise the reallocation may only result in a randomized block already being used by others. But LTE lacks the exploration of one important concept called RRM (Radio Resource Management) [17] which means LTE has lesser control of co-channel interference and transmission parameters.

The Generations of development of mobile networking is clearly marked with sophistication of technologies, and environmental utilization like modulations to accommodate more signals in a single channel. The basis of service has been the assurance of QoS and the basis of QoS is ensuring less interference. However, as need grows stronger, the quench for bandwidth also grows. That is why the generations of development will keep rolling with superior promises of sophistication.

The future development of Internet communication will be largely based on wireless access to and from heterogeneous devices. The current $4 \mathrm{G}$ network support is using the promising LTE technology which is just at its inception and being practiced only in some minor parts of the world. With passage of time more devices will be utilizing $4 \mathrm{G}$ and many issues are likely to come up.

The worldwide consumption of data is going to be far more than what is being consumed now. By 2016 about $71 \%$ of network traffic will be video traffic [18] and smartphones will count over 100 million with a monthly bandwidth consumption of over 1 GB [19]. Such enormous consumption exceeds the capacity of 4G/WiMax/LTE [20].

The major concern will be infrastructure development to meet upcoming standards with focus on traffic accommodation and interference management. Research issues like femtocells, high altitude platform, and cognitive radio technology are some of the many approaches to such developments. The demand for high-speed internet access including livestreaming and video-on-move with less delay, jitter, improved QoS, less interference will be the drives for $5 \mathrm{G}$ developments in future.

\section{5G TECHNOLOGIES}

\subsection{Femtocells}

A Femtocell is an economical base-station having powersaver and easy-deployable features with a range of some tens of meters [21]. It is designed to be able to optimize and configure itself dynamically with a Plug-and-Play deployment ease. On the other side, UEs are required to search for femtocells automatically [22].

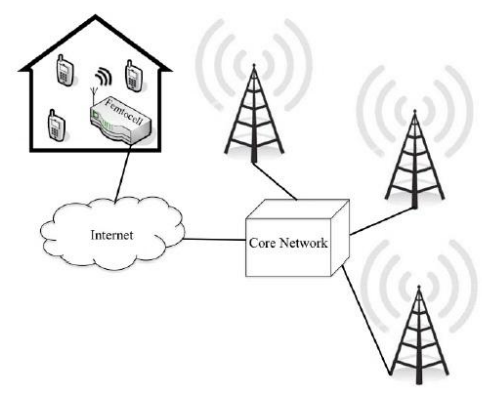

Fig 3: Femtocell in cellular network [26]

Femtocells are smaller cells installed indoors while they connect to the network using DSL backhaul; it increases user throughput from shorter ranges, increases system capacity through spatial reuse, improves operators' coverage and service quality with low cost and load-balancing by trafficoffloading from macrocells to femtocells [23]. To achieve the full benefits, the femtocells need to use the same technology as macrocells, and also needs to mitigate interference in between. But on the other hand, using the same spectrum causes interference between them. FFR (Fractional Frequency Reuse) - which is a dynamic frequency allocation mechanism - is proposed to mitigate the interference [24]. The key to balancing between femtocells and macrocells are mobility, load, and power.

Kulkarni et al. proposes some design considerations for femtocell deployment [25]. The premise of deployment, applications and usage patterns, resource allocation, detection of UE interference and the device interfering with it, and a good plan for negotiating resources to overcome interference are some of the proposed design considerations for femtocells.

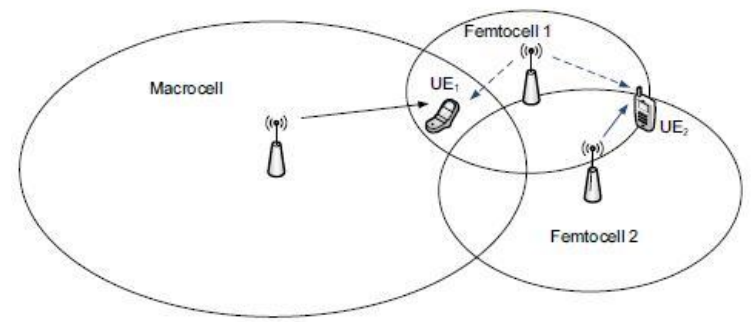

Fig 4: Femtocell and Macrocell architecture and interference [25]

Femtocells are utilized with FAPs (Femtocell Access Points), which improve network capacity by reusing the frequency in their smaller coverage areas [25]. The main advantage of femtocells is they can bridge the dead zones of an inside premise where macrocell cannot penetrate. The cells need to minimize mobility issues like the take-over of macrocells for the roaming users who move between femtocells. Traffic offloading from macrocell to femtocell also benefits the macrocell performance and reduces operating expenses.

One of the technologies used by FemtoCells is OFDMA which enables easy air-interface. This also brings into consideration the resource management in the perspective of air-interference. [23] proposed some mechanisms for efficient resource management $-i$ ) isolating resources in frequency domain, ii) intelligent separation between link adaptation and resource allocation, iii) client scheduling in same frame, iv) 
fair resource allocation with high utilization and low overhead.

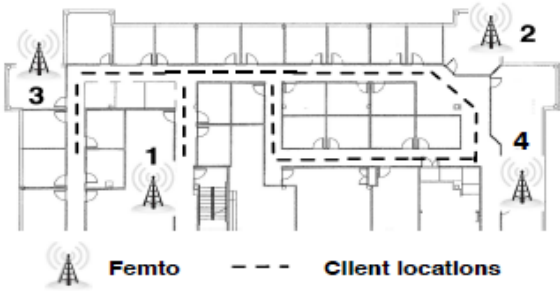

Fig 5: FemtoCells in deployment [23]

After powering up the femtocells need to discover network operator and register, then they receive configuration parameters from operator. One major disadvantage of femtocells is they mostly operate on the same frequency and therefore vulnerable to interference. Femto forum suggests three mechanisms for mitigation of interferences [28]. The first one is optimal transmission power - not to affect the neighboring cells but enough for the coverage area. The second one is optimal receiving power - not to allow neighboring cells to anchor in the femtocell's coverage area. The third one is applying a threshold uplink power to the UE so that if no neighboring femtocell is accessible, then the UE can attach to the nearest macrocell rather than transmitting high power to reach a femtocell.

The self-configuration mechanism of femtocell means essentially the resource negotiation which can be brought out by two types of deployment [28]. One is called dedicated channel deployment which dedicates portion of spectrum to femtocells, and the rest to macrocells. The other one is called co-channel deployment which deploys the same spectrum to both cells. The former one is costly due to dedicated spectrum, but it can differentiate femtocell interference from macrocell interference, thereby increases macrocell efficiency. The second one cost-effective but cannot differentiate the interferences. It may be mentioned that macrocell users have higher priority than femtocell users.

Femtocell employs self-organized CAT (Coordinated Access Technology) which consumes low power; it is less costly, and enhances cell capacity and coverage [17:160]. But they have their own interference problems to which some distributed mechanisms are proposed by some studies [29]. It is observed that femtocells are vulnerable to attacks on user identity, data, protocols and configurations; eventually still being deficient to fulfil 3GPP security measures [30]. They are also susceptible to spatial interferences [31].

Femtocell is a growing concern for the next generation mobile Internet connectivity. The higher bandwidth offered by femtocells for inside premises is an important element to consider for the speedier Internet development [24]. The loadbalancing and traffic-offloading are two important aspects of femtocells. On the other hand innovative measures are also being proposed for the interference mitigation. With all such offers and effectiveness femtocells is supposed to be a promising feature for future Internetworking.

\subsection{High Altitude Platforms}

HAP (High Altitude Platform) is one other new innovative idea for mobile networking. These can be thought of as some lowered satellites or very high radio mast, and offers features of both the systems [9].
Elsafrawey et al. provides some clear definitions of HAP [10]. This is actually a $(150 \mathrm{~m} \times 40 \mathrm{~m})$ airship located in the stratosphere between 17 and $30 \mathrm{~km}$ from ground. The airship operates by highly-pressurized helium or solar power. It is equipped with telecommunication systems to support an entire region and doesn't require additional stations for service. Statistically 250 HAPs over 250 crowded cities are supposed to serve over $80 \%$ of world population [10].

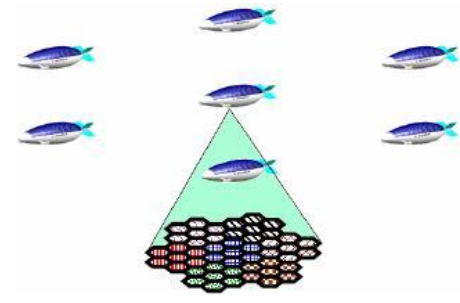

Fig 6: Generic structure of HAP and terrestrial system [10]

HAP is specially a cost-effective solution for rural and maritime regions, and the promising applications are cellular networking, broadband LMDS (local multipoint distribution system), and Internet and ISDN [10]. Such systems are flexible with mobility option and technology upgrades, and can be connected to neighboring HAPs with help of intermediate hop stations or links. HAPs have a similar counterpart named HAVE (High Altitude Very long Endurance) which is a similar implementation for vehicles specifically.

Likitthanasate et al., mentioned the possibility of coexistence of HAP and WiMAX with BPSK (Binary Phase-Shift Keying), which is a digital modulation technique that transmits data by modulating the reference signal and uses two phases for modulating [12]. The architectural constraint

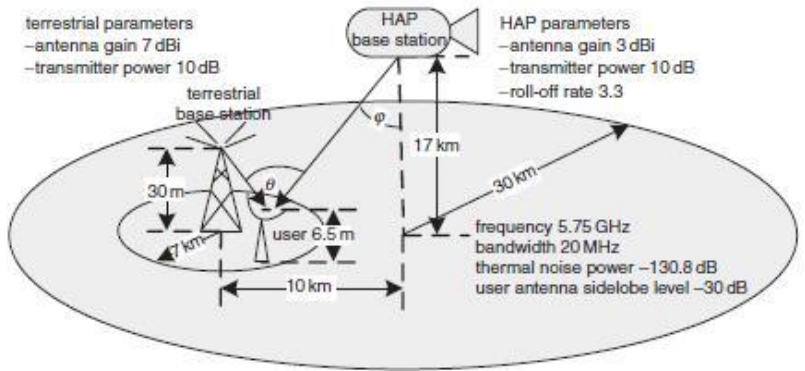

Fig 7: Coexistence of HAP and terrestrial base station - at a more specific glance [12]

for this is that the beam-width of the user-antenna needs to have a special angle of $\angle 30^{\circ}$ to be able to use both HAP and terrestrial users (WiMAX) at the same time without the need for other sophistications. If wider beam-width is required, then other concerns like spectrum separation and licensing, and management of power and spectrum need to be addressed.

As obvious from the generic structure of HAPs and terrestrial systems, the radius of service of HAPs largely depends on the height of the station. Taha-Ahmed et al. studied the effect of HSDPA over HAP with regards to height of HAP, radius of cell and beaming direction of cell at $0^{\circ}$ and $30^{\circ}$ [9]. They found that, at a given modulation scheme, the effective range of HAP is less in urban zone than rural zone. Using $80 \%$ of base-station power at the highest modulation of ( $3 / 4$ 16QAM) three users can be connected with HSDPA in rural areas while 
only one user can connect to HSDPA in urban zone [9]. The problems associated with HAPs are mostly related to aerial atmosphere. They face problems of free space loss due to air and moisture [32]. So the expected services from HAPs are variable with regards to aerial condition.

HAP is an inexpensive alternative to existing terrestrial and satellite systems with capacity improvements [9]. In fact they can relieve the satellites to a greater extent from such communication activities and they can prove to be more effective with regards to cost and service. Researches need to be undertaken to find measures for tweaking a HAP because of aerial interference. HAPs are an economic and effective consideration for the next generation of mobile Internetworking.

\subsection{Cognitive Radio}

It needs to be clarified that the problem with sharing spectrum is not scarcity, but the way it is used [33]. According to FCC (Federal Communications Commission), about $70 \%$ of the officially allocated spectrum sits idle at several times of a day [33]. This is where a cognitive radio can come to a relief. A cognitive radio is a mechanism that can try to detect unused frequencies in the spectrum and try to utilize that optimistically with a view to utilizing the spectrum to its full potential. Such a radio network is called CRN (Cognitive Radio Network)

There are two kinds of spectrum users - PU (Primary User) who are non-cognitive, and SU (Secondary User) who are cognitive [34]. The negotiation between the original owner of the bandwidth and the optimistic device can be accomplished through a connected channel between them. But this in turn occupies a channel whereas the ultimate goal is to exploit unused channels.

There are two types of CR with regards to spectrum usage one is underlay CR, the other is overlay CR. Underlay CR permits coexistence of primary and secondary transmissions only if the primary user experiences interference below a defined threshold. The overlay CR uses dirty paper coding at the secondary transmitter to mitigate interference, but allows concurrent primary and secondary transmissions as well [34:1]. Overlay CR uses unused spectrum, on the contrary, underlay $\mathrm{CR}$ uses underused spectrum. Interweave $\mathrm{CR}$ is weaving a wider-band $\mathrm{CR}$ with narrow-band $\mathrm{CR}$ [52] that uses opportunistic communication and spectrum holes [34:1].

CRN works with spectrum access technologies, environment sensing, adaptation, intelligent radio functions and smart antennas. Adaptation and flexibility are the main concerns of CRN. Actually it is the sporadic use of licensed spectrum that leads the thinking of CRN [36].

As a minimal requirement, a CRN should consider cross-layer architecture with several QoS service abilities, adaptation to fluctuating spectrum and learning optimal bandwidth allocation, and spectrum molding at transmitter-end to result in lesser interference [37].

Zheng suggests utilizing game theory instead of negotiation [33], which in this scenario would suggest observing the transmission of neighbors and make its own decision. She compares this with trafficking system where a driver in a crowded lane needs to observe others and carefully switch to a less crowded lane so that the car doesn't collide with others. But applying game theory to CRN incurs signaling overhead and defers scalability [38]. This is because it constraints that each user possesses global information about everybody in the specific network which is certainly an unexpected signaling overhead.

Dynamic Spectrum Access is a research interest which basically constitutes of finding out holes in spectrum (unused spectrum). These frequency voids (i.e. spectrum holes) vary with time and location. Hence user location and faster handover between cells of different networks are utilizing spectrum holes [37].

Arienzo classifies some CRN-based applications that might be developed in near future [37]. Smart Home Applications providing lots of functionality from multimedia to elderly support, Surveillance Sensor Networks providing real-time data and preventing criminal actions and violation of laws, Smart Transportation Applications for transportation

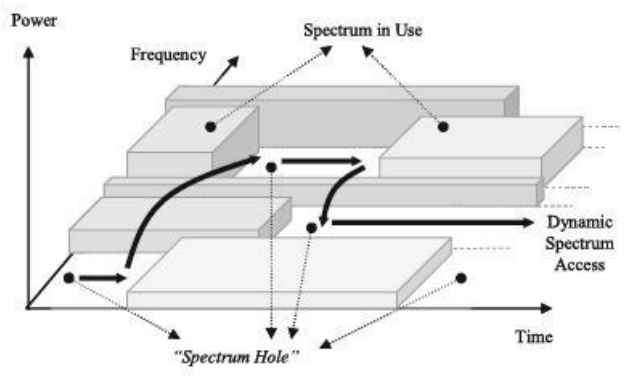

Fig 8: Spectrum-hole concept [39]

efficiency, Advanced Health Care applications where patients carry sensors in their body which updates the patient database in real-time and signals any emergency, Industrial Process Control for industrial automation with machine vision, Smart Office Applications boosting up various official works including tele-working to reduce physical stress are some of the applications foreseen with regards to CRN.

As for CRN enhancements opportunities, Kocks et al. proposes a signal processing platform with FPGA utilization concerning scalability, and modularity [40]. Alongside, Goldsmith et al. represents $\mathrm{CR}$ as an intelligent wireless communication device that interweave, underlay, and overlay [41]. He also stressed on a priori rules on spectrum usage, as well as side knowledge on activity, channel, codebooks, and/or messages, non-cognitive nodes. There might be researches on minimizing the forced-termination probability [31] to achieve a continuous connection through channels. There are opportunities for algorithmic developments. To name one, a broadcasting algorithm is devised by Ertuğrul \& Buzluca [42] named Primary User Aware, Request for Message. Broadcasting is a required in any ad hoc network for various purposes, and same is true for CRAHNs (Cognitive Radio Ad Hoc Networks). The algorithm works with invitation to all PUs in a channel with less PU activity. Any node that misses a message requests the message from source. The algorithm utilizes a counting mechanism to avoid unnecessary message broadcasting.

The present problems with CRN are mostly associated with channel utilization. The selection of frequency channel and transmission power at the same time might create problems [43]. There is obvious chance for false channel detection. Such false alarm and probability of misdetection can be overcome with increasing listening stage for sensing algorithms [44].

A CRN is an intelligent concept of utilizing the existing spectrum of the environment to its full potential. Utilizing the 
idle spectrum in the air with proper negotiation between privileged and non-privileged users is the main concept of the CRN. New methods and concepts are being developed to solve or at least to reduce the inherent problem of CRN regarding spectrum mismanagement. Many newer research concepts are on way to work with. With proper handling, a CRN can be a potential candidate to consider for next generation mobile network.

\section{CRITICAL EVALUATION}

Femtocells, HAPs and CRNs are three separate approaches to accommodate the tremendous speeding and QoS requirements for the coming generation of Internetworking. While femtocells are ground-based stations to assist macrocells, the HAPs are airships located high in the air to assist rural and maritime Internet connectivity, while $\mathrm{CRN}$ is the clever utilization of distributed and ubiquitous, but idle spectrum throughout the air.

Femtocells are indoor base-stations with shorter ranges; these directly utilize the OFDMA mobile technology. HAPs are located in the air and they work as intermediary satellites between base stations and space satellites; specifically they don't linger on any specific technology like OFDMA, but researches are being done on the effect of HSDPA over HAP [9]. CRNs mostly utilize the spectrum access technologies like CRAHNs and dynamic spectrum access; and as obvious from the purpose, CRNs don't have any specific location they are the ubiquitous spectrum that remains everywhere.

The range of femtocells is typically some 10's of meters [21], the range of HAPs are typically $30 \mathrm{~km} \mathrm{[12].} \mathrm{There} \mathrm{is} \mathrm{no} \mathrm{range}$ consideration for a CRN as this is a spectrum access technology that doesn't talk about ranges.

Each of these technologies has its own utilization and benefits. Femtocells assist macrocells by taking over responsibilities, HAPs can assist the terrestrial and satellite systems working as intermediary assistant, $\mathrm{CRNs}$ can assist connectivity by utilizing the idle environment to its full potential. Similarly every one of them has its own problems. Femtocells have spatial interference problems [31], and also vulnerable to user identity, data, protocols and configurations [30]. HAPs suffer from free space loss due to air and moisture [32]. CRNs have problems with false channel detection and transmission [43].

OFDMA is a chosen technology between the standards for current communication. LTE provides a re-engineering of OFDMA to provide speedier traffic management. Uninterrupted service is provided with Interference management and QoS services, where QoS can further be translated to QoE to take the user experience to further satisfactory level.

There can be a mix of existing efficient technologies with these advanced technologies. For example, HSPA, EVDO, WiMAX and LTE - all are spectrally efficient and data-caring standards [26]. We have technological advancements with regards to aerodynamics, avionics, and electronics [45]. Access network selection mechanisms are mobile deviceoriented at the moment. But such mechanism fails to effectively comprehend the networking environment at the moment and incurs power cost with access network selection with multiple scans [46]. Such issues need to be sewed with the deployment of the discussed technologies to take those steps ahead and make a smooth passage for future networking in the next generation.

\section{FUTURE RESEARCHES}

Future Internetworking for the next generation will heavily rely on QoS-aware, QoS-guranteeing protocols and algorithms. At the moment, interference management seems to be one of the major issues with wireless network. Proposals have been made for improvising the existing protocols. For example, adaptive scheduling for LTE is proposed by Ahmed et al. [17], cross-layer design and the multi-hop communication is proposed by Arienzo [37], Genetic algorithms for communication is proposed by Canales \& Gállego [43], femtocell coverage algorithm is proposed by Ho et al. [47].

The improvisations not only result in new concepts, but some also device clever maneuver of the transmitting data. For example, Bao et al. proposes a context-aware compression mechanism to reduce the image traffic [48]. An image is partitioned into main portion and less important portions. On demand, the main content is transferred and the receiver inserts an arbitrary image from the specified domain to approximate the original image. Their proposition is based on some hypotheses like parts of image are less valuable, human perception to important parts of the image are mostly similar, and background removal decreases image size, hence speeds up the process.

Wide channel bandwidth and OFDMA/OFDM-based physical layer are the chosen technologies for $4 \mathrm{G}$ for high data rate and better QoS [32]. But power-consuming OFDMA/OFDM consumes the cell power more quickly. This brings in considerations for smaller cell sizes, which in turn imposes cost of renovation and sophistication. This leads to research opportunities for power-saving cell design, and accommodating heterogeneous technologies in cells.

To accommodate and load-balance heterogeneous technologies like LTE, GPRS, WiMAX, and WiFi the 3GPP proposes an all-IP multiple access core network [46]. It is well apprehended that problems with collaboration and coexistence of such disparate technologies will eventually open opportunities for further sophistication to this all-IP network.

Bhaumik et al. highlights on energy consumption on cell sizes where cell-size can be dynamically adjusted to obtain optimal energy consumption [49]. The optimal cell size depends on base station, traffic and data rates.

From among other research issues, there are ubiquitous computing and DAWN (Dynamic Ad hoc Wireless Networking). Ubiquitous computing is the computing that takes place everywhere pervasively, and not confined to the desktop computer only. This is more an abstract thinking than any technical deployment. Other terminologies that are used synonymously are pervasive computing, and $\mathrm{ABC}$ (ActivityBased Computing). For ubiquitous computing, there might be research interests in location management which involves position, nearest neighbour, and range-related concerns [50].

\section{CONCLUSION}

Mobile networking is going to be the most-utilized communication means in near future. In fact except for the networks where the infrastructure is already set up for mainframe-based or high-speed computing environment, the other communication devices have already started to switch to mobile networking. For example, personal laptops are mostly using dongles for wireless Internet connections. Over 1 billion mobile devices are being sold each year [51] among which the significant number is capable of Internet communication. High-speed Internet access while on move is going to be the 
normal demand from users even from technological point-ofview these are sophisticated issues to be handled with a blend of technologies, algorithms, protocols. This aims at better QoS with reduced interference. The novel approaches like femtocells, High Altitude Platforms and Cognitive Radio may be a 'fantastic three' combination for the next generation mobile Internet. But to remember, they are some of the many techniques to technically approach to meet the demands as well as to effectively address the existing limitations. The footsteps for $5 \mathrm{G}$ has just commenced and yet to standardize, but the technological researches have enthusiastically commenced with such promising and innovative technologies.

\section{REFERENCES}

[1]. Leu, S.J., and Chang, R.S. (2003). Integrated service mobile internet: RSVP over mobile IPv4\&6. Mobile Networks and Applications, Volume 8 Issue 6.

[2]. Giordano, S., Salsano, S., Bergue, S.V., Ventre, G., and Giannakopoulos D. (2003). Advanced QoS Provisioning in IP Networks: the European Premium IP Projects. IEEE Com. Mag. January 2003.

[3]. Leyden, J. (2011). World shrugs as IPv4 addresses finally exhausted. Available at: http://www.theregister.co.uk/2011/02/02/ipv4_exhaustio $\mathrm{n} /$ [Accessed on 13 May 2012].

[4]. Perkins, C. (1996). Ed., IP mobility support. IETF RFC 2002 (October 1996).

[5]. Perkins, C. (1998). Mobile IP: Design Principles and Practice. Addison-Wesley/Longman, Reading, MA.

[6]. Perkins, C. (1998). Mobile networking through mobile $I P$. IEEE Internet Computing (January-February 1998) Pages 58-69.

[7]. Clincy, V.A., and Mudiraj, P. (2006). The future leading mobility protocol: mobile IPv4 OR Mobile IPv6? Journal of Computing Sciences in Colleges, Volume 22 Issue 1, Pages 197-203.

[8]. Johnson, B.D., and Perkins, C.E. (2001). Mobility support in IPv6. Internet Draft (July 2001).

[9]. Taha-Ahmed, B., Calvo-Ramón, M., and De Haro-Ariet, L. (2007). UMTS-HSDPA in High Altitude Platforms (HAPs) Communications. Turkish Journal of Electrical Engineering \& Computer Sciences, 2007, Vol. 15 Issue 1, Pages 105-112.

[10].Elsafrawey, A.S., and Eldolil, S.A. (2007). Performance evaluation for integrated high altitude platformsterrestrial general packet radio service system. October 2007 WMuNeP '07: Proceedings of the 3rd ACM workshop on Wireless multimedia networking and performance modeling. Publisher: ACM.

[11].Segan, S. (2012). $3 G$ Vs. 4G: What's the Difference? PCMag.com. Available at: http://www.pcmag.com/article2/0,2817,2399984,00.asp [Accessed on 22 June 2012].

[12].Likitthanasate, P., Grace, D., and Mitchell, P.D. (2005). Coexistence performance of high altitude platform and terrestrial systems sharing a common downlink WiMAX frequency band. Electronics Letters, 7/21/2005, Vol. 41 Issue 15 , pages $1-2,2 \mathrm{p}$.

[13].Baldo, N., Miozzo, M., Esteso, M.R., Guerrero, J.N. (2011). An open source product-oriented LTE network simulator based on ns-3. October 2011, MSWiM '11: Proceedings of the 14th ACM international conference on Modeling, analysis and simulation of wireless and mobile systems. Publisher: ACM.

[14].Kittipiyakul, S., and Javidi, T. (2004). Subcarrier Allocation in OFDMA Systems: Beyond water-filling. Signals, Systems, and Computers, 2004.

[15].Quek, T., Lei, Z., and Sun, S. (2009). Adaptive Interference Coordination in Multi-cell OFDMA Systems. IEEE PIMRC, 2009.

[16].Kulkarni, P., Chin, W.H., Farnham, T. (2010). Radio resource management considerations for LTE Femto cells. January 2010, SIGCOMM Computer Communication Review, Volume 40 Issue 1. Publisher: ACM.

[17].Ahmed, I., Badia, L., Baldo, N., and Miozzo, M. (2011). Design of a unified multimedia-aware framework for resource allocation in LTE femtocells. October 2011 MobiWac '11: Proceedings of the 9th ACM international symposium on Mobility management and wireless access. Publisher: ACM.

[18].CISCO (2012a). Cisco Visual Networking Index (VNI): Global Mobile Data Traffic Forecast, 2011-2016 [Visual Networking Index]. Available online at: http://www.cisco.com/en/US/solutions/collateral/ns341/n s525/ns537/ns705/ns827/VNI-Forecast_QA.html [Accessed 29 April 2012].

[19].CISCO (2012b). Cisco Visual Networking Index: Global Mobile Data Traffic Forecast Update, 2011-2016 [Visual Networking Index]. Available online at: http://www.cisco.com/en/US/solutions/collateral/ns341/n s525/ns537/ns705/ns827/white_paper_c11-520862.html [Accessed 29 April 2012].

[20].Rysavy Research (2010). Mobile Broadband Capacity Constraints and the Need for Optimization.

[21].Chandrasekhar, V., Andrews, J., and Gatherer, A. (2008). Femtocell networks: a survey. Communications Magazine, IEEE, 46 (9). Pages 59-67.

[22].Lee, P., Jeong, J., Kim, Y.H., Shin, J. (2010). Physical cell identity reservation for $3 G P P$ LTE femtocell. January 2010 ICUIMC '10: Proceedings of the 4th International Conference on Uniquitous Information Management and Communication. Publisher: ACM.

[23].Arslan, M.Y., Yoon, J., Sundaresan, K., Krishnamurthy, S.V., and Banerjee, S. (2011). FERMI: a femtocell resource management system forinterference mitigation in OFDMA networks. September 2011 MobiCom '11: Proceedings of the 17th annual international conference on Mobile computing and networking. Publisher: ACM.

[24].Lee, S., Ahn, C., Cho, T., Park, J., Shin, J. (2012). Dynamic frequency allocation of femtocells for guaranteed macrocell throughput in OFDMA networks. February 2012, ICUIMC '12: Proceedings of the 6th International Conference on Ubiquitous Information Management and Communication. Publisher: ACM.

[25].Kulkarni, P., Chin, W.H., Farnham, T. (2010). Radio resource management considerations for LTE Femto cells. January 2010, SIGCOMM Computer Communication Review. Volume 40 Issue 1. Publisher: ACM. 
[26].Mungan, D., Ferazoglu, G., Sunay, O., Civanlar, R., and Kaytaz, B. (2011). Advantages of application-based scheduling for indoor femtocells. October 2011, WMuNeP '11: Proceedings of the 6th ACM workshop on Wireless multimedia networking and computing.

[27].Teracom Training Institute Ltd (2006). Teracom Tutorial: OSI 7-Layer Model [online] http://www.telecommunications-tutorials.com/tutorialOSI-7-layer-model.htm [Accessed 17 May 2012].

[28].Femto Forum White Paper (2010). Interference Management in UMTS Femto Cells. http://www.smallcellforum.org/smallcellforum_resources /pdfsend01.php?file $=009 \% 20$ Interference $\% 20$ Manageme nt\%20in\%20UMTS\%20femtocells.pdf, Feb. 2010. Accessed 3 May 2012.

[29].Sundaresan, K., and Rangarajan, S. (2009). Efficient Resource Management in OFDMA Femto Cells. ACM MobiHoc, May 2009.

[30].Borgaonkar, R., Redon, K., Seifert, J.P. (2011). Security analysis of a femtocell device. November 2011 SIN '11: Proceedings of the 4th international conference on Security of information and networks. Publisher: ACM.

[31].Chen, C.W., Wang, C.Y., Chao, S.L., Wei, H.Y. (2010). DANCE: a game-theoretical femtocell channel exchange mechanism. July 2010, SIGMOBILE Mobile Computing and Communications Review, Volume 14 Issue 1. Publisher: ACM.

[32].Imran, A., Shateri, M., and Tafazolli, R. (2009). On the comparison of performance, capacity and economics of terrestrial base station and high altitude platform based deployment of 4G. October 2009 PE-WASUN '09: Proceedings of the 6th ACM symposium on Performance evaluation of wireless ad hoc, sensor, and ubiquitous networks. Publisher: ACM.

[33].Savage, N. (2006). Cognitive Radio. Technology Review, Mar/Apr2006, Vol. 109 Issue 1, Pages 61-62.

[34].Re, E.D., Pucci, R., Ronga, L.S. (2011). Energy efficient resource allocation game for cognitive radio. October 2011 CogART '11: Proceedings of the 4th International Conference on Cognitive Radio and Advanced Spectrum Management. Publisher: ACM.

[35].Srinivasa, S., and Jafar, S.A. (2007). The Throughput Potential of Cognitive Radio: A Theoretical Perspective. IEEE Communications Magazine, May 2007.

[36].Gad, M.M., El-Khamy, M., Mouftah, H.T. (2011). Dual band connectivity of cognitive radio networks. October 2011 CogART '11: Proceedings of the 4th International Conference on Cognitive Radio and Advanced Spectrum Management. Publisher: ACM.

[37]. Arienzo, L. (2011). Internet of networks: is the future internet a cognitive radio application? October 2011 CogART 11: Proceedings of the 4th International Conference on Cognitive Radio and Advanced Spectrum Management. Publisher: ACM.

[38].Komali, R.S., Mackenzie, A.B., and Mahonen, P. (2009). On selfishness, local information, and network optimality: a topology control example. Proc. ICCCN’09, San Francisco, CA, USA, August 2009.
[39].Akyildiz, I.F., Lee, W.Y., Chowdhury, K.R. (2009). CRAHNs: Cognitive radio ad hoc networks. Ad Hoc Networks 7 (2009), pages 810-836.

[40].Kocks, C., Viessmann, A., Skrebtsov, A., Bruck, G.H., Jung, P. (2011). Concept and design of a cognitive radio prototyping platform. October 2011 CogART '11: Proceedings of the 4th International Conference on Cognitive Radio and Advanced Spectrum Management.

[41].Goldsmith, A., Jafar, S.A., and Srinivasa, S. (2009). Breaking spectrum gridlock with cognitive radios: An information theoretic perspective. Proc. of the IEEE, vol. 97, no. 5, Pages 894-914, May 2009.

[42].Ertuğrul, O., Buzluca, F. (2011). An efficient broadcasting scheme for cognitive radio ad hoc networks. October 2011 CogART '11: Proceedings of the 4th International Conference on Cognitive Radio and Advanced Spectrum Management. Publisher: ACM.

[43].Canales, M., and Gállego, J.R. (2010). Potential game for joint channel and power allocation in cognitive radio networks. Electronics Letters, 11/25/2010, Vol. 46 Issue 24, Pages 1632-1634.

[44].Cardenas-Juarez, M., Ghogho, M. (2011). Spectrum sensing and data transmission trade-off in cognitive radio under outage constraints. Electronics Letters, 3/31/2011, Vol. 47 Issue 7, pages 469-471, 3p, 2 Graphs.

[45].Karapantazis, S., Pavlidou, F.-N., (2005). The role of high altitude platforms in beyond $3 G$ networks. Wireless Communications, IEEE , vol.12, no.6, Pages 33-41, Dec. 2005.

[46].Corici, M., Fiedler, J., Magedanz, T., Vingarzan, D. (2011). Access Network Discovery and Selection in the Future Wireless Communication. June 2011, Mobile Networks and Applications, Volume 16 Issue 3.

[47].Ho, L.T.W., Ashraf, I., and Claussen, H. (2010). Evolving femtocell coverage optimization algorithms using genetic programming. In Personal, Indoor and Mobile Radio Communications, 2009 IEEE 20th International Symposium on, pages 2132-2136. IEEE, 2010.

[48].Bao, X., Narayan, T., Sani, A.A., Richter, W., Choudhury, R.R., Zhong, L., Satyanarayanan, M. (2011). The case for context-aware compression. March 2011, HotMobile 11: Proceedings of the 12th Workshop on Mobile Computing Systems and Applications.

[49].Bhaumik, S., Narlikar, G., Chattopadhyay, S., Kanugovi, S. (2010). Breathe to stay cool: adjusting cell sizes to reduce energy consumption. August 2010, Green Networking '10: Proceedings of the first ACM SIGCOMM workshop on Green networking.

[50].Becker, C., Dürr, F. (2005). On location models for ubiquitous computing. January 2005 Personal and Ubiquitous Computing, Volume 9 Issue 1.

[51].Cellular-News (2007). Over 1 Billion Phones Sold Last Year. http://www.cellular-news.com/story/21622.php [Accessed 28 May 2012]

[52].Chang, C.W. (2010). An Interweave Cognitive Radio System Based on the Hierarchical 2D-Spread MC-DSCDMA. Vehicular Technology Conference Fall (VTC 2010-Fall), 2010 IEEE $72^{\text {nd }}$, Pages 1-5. 